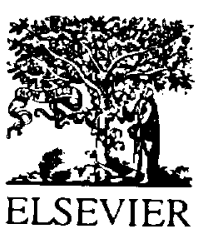

Earth and Planetary Science Letters 131 (1995) 27-39

\title{
Chondrule formation, metamorphism, brecciation, an important new primary chondrule group, and the classification of chondrules
}

\author{
Derek W.G. Sears, Huang Shaoxiong, Paul H. Benoit \\ Cosmochemistr. Group, Department of Chemistry and Biochemistry, Unicersiry of Arkansas, Falctterille. AR 72701. USA \\ Received 17 May 1994: accepted after revision 9 January 1995
}

\begin{abstract}
The recently proposed compositional classification scheme for meteoritic chondrules divides the chondrules into groups depending on the composition of their two major phases, olivine (or pyroxene) and the mesostasis, both of which are genetically important. The scheme is here applied to discussions of three topics: the petrographic classification of Roosevelt County 075 (the least-metamorphosed $\mathrm{H}$ chondrite known), brecciation (an extremely important and ubiquitous process probably experienced by $>40 \%$ of all unequilibrated ordinary chondrites). and the group A5 chondrules in the least metamorphosed ordinary chondrites which have many similarities to primary formation properties of possible to determine the petrographic type of $\mathrm{RC}$ and the effects of metamorphism on the entire assemblage it is applied to individual chan scheme can be especially suitable for identified by previous techniques and which app, the new scheme has revealed the existence of chondrules not (but unlike group B1 chondrules) the primitich appear to be extremely important. Like group Al and A2 chondrules group $\mathrm{A} 1$ and $\mathrm{A} 2$ chondrules (and like grouve group A5 chondrules did not supercool during formation. but unlike formation. It is concluded that formation and history of chondrules and
\end{abstract}

\section{Introduction}

By virtue of their great age, solar composition, unusual isotopic properties and relatively unaltered textures chondrites provide unique insights into early solar system processes [1]. One of the major components of chondrites are the chondrules [2], whose presence implies that pulse heating was a common phenomenon in the early solar system $[1,3]$. However, the origin of chon- drules, and their possible relevance to the formation of the major chondrite classes, has proved highly contentious [4,5]. Some of the controversy is due to the great diversity and complexity of chondrules. The classification of chondrules is therefore an essential step in understanding this complexity and the questions surrounding these objects. As part of our efforts in this direction we recently proposed a new classification scheme for meteoritic chondrules $[6,7]$. 
In a subject plagued with a great many classification schemes it is essential to show restraint in proposing yet another. We certainly endorse Wood's comment that bad classification schemes can obscure relationships and even cloud observations [8]. Our reasons for proposing a new scheme were prompted by a very pragmatic issue: In attempting to understand the thermoluminescence (TL) and cathodoluminescence (CL) properties of chondrules we needed a way of quickly sorting them into meaningful groups, each with reasonably uniform properties, independent of the host meteorite. We found that with existing schemes an uncomfortable fraction seemed to be 'anomalous'. Our new scheme for chondrule classification relies on the compositions of the two major components, the olivine grains (or pyroxene grains in the very few chondrules without olivine) and the surrounding mesostasis. This enables classification of $>95 \%$ of the chondrules in a meteorite. However, having developed the new scheme we quickly realized that there were several significant advantages to the scheme which are of a more fundamental kind. This is because both the olivine (or pyroxene) and the mesostasis of chondrules display major compositional trends which reflect differences in original formation processes and the subsequent overprint of metamorphism.

In a recent paper Scott et al. [9] pointed out several ways in which our compositional classification scheme could be improved, although they favored the retention of their previous system. They did not discuss the main strengths of the new scheme which are that, unlike its predecessors, it is simple, comprehensive and, most importantly, lacks assumptions about the original nature of a given chondrule and its subsequent alteration. In this paper we briefly summarize our chondrule classification scheme, including some refinements added to incorporate the improvements suggested by various researchers. We then discuss three applications of the new scheme which demonstrate its utility. Throughout this paper we attempt to distinguish between (1) the mechanical (but very important) procedure of classifying chondrules based on their mineral chemistry and (2) interpretations derived from these data concerning the processes of metamorphism, brecciation and chondrule formation.

\subsection{Summary of the compositional classification of chondrules and comparison with previous schemes}

We define eight chondrule classes in terms of the compositional boundaries for the chondrule olivines shown in Fig. 1 and the compositional boundaries for the mesostasis shown in Fig. 2. We do this because (1) these are normally the two major components of the chondrules and (2) it has been shown that the chemistry of both are

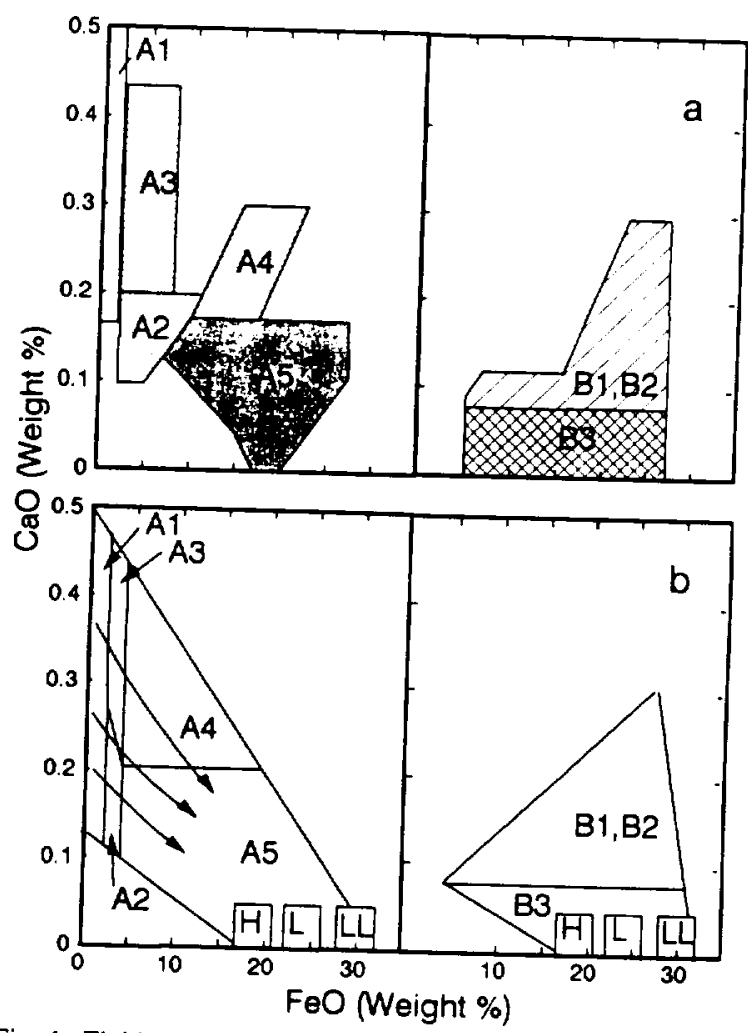

Fig. 1. Fields used to define compositional classes for chondrules using the $\mathrm{CaO}-\mathrm{FeO}$ plot. The fields proposed by Sears et al. and DeHart et al. [6,7] are shown (a) together with (b) slightly simplified, and perhaps more realistic, versions. Also shown are the fields for equilibrated $\mathrm{H}, \mathrm{L}$ and $\mathrm{LL}$ chondrites. Because chondrules display such large compositional ranges it would be meaningless to define separate fields for chondrules from unequilibrated $\mathrm{H}, \mathrm{L}$ and $\mathrm{LL}$ chondrites, but minor modification might be required in order to apply these fields to enstatite and carbonaceous chondrites. 
of considerable importance in deciphering chondrule history. Rather than document the painstaking and slow process by which chondrule classification has evolved we have attempted to explain how previous chondrule classification schemes relate to the present proposals as footnotes to Table 1 . The present scheme picks up on all of the previously recognized groups, and adds others, but does so in a simple way and in a way that is not compromised by metamorphism and brecciation.

The redox state of the environment and loss of volatiles during chondrule formation have both been discussed by many authors (e.g., $[6,7,10-15]$ ). Laboratory heating experiments and thermodynamic calculations show that considerable loss of volatiles and reduction of $\mathrm{FeO}$ to $\mathrm{Fe}$ should occur at or near the melting point if chondrules were heated in a gas of even approximately cosmic composition, and yet many chondrules show little or no volatile loss or reduction (e.g., [12]). There have been a great many ideas proposed whereby chondrules could be heated to near the melting point in order to obtain their observed textures and yet not undergo compositional changes. However, McSween [16] showed that a significant number of chondrules in carbonaceous chondrites did appear to be both reduced and poor in volatiles. These were termed 'type l', while the term 'type II' was reserved for chondrules which were not volatile-poor or reduced. Scott and Tay- lor [17] showed that these two distinctions could be recognized among the chondrules of ordinary chondrites, and they further distinguished between 'type IA' and 'type IB' chondrules, the latter meaning FeO-poor chondrules in which olivine was poikilitically enclosed in pyroxene. More recently, Jones [18,19] introduced 'type IAB' for FeO-poor pyroxene-rich chondrules. Subdivision of the type II chondrules along similar lines is a likely future development.

Chondrule mesostasis is important not only because it is the major depository of the volatiles but also because it provides unique insights into the crystallization history of the chondrules [2023]. Some chondrules (which are termed groups A1, A2 and A5 in the compositional classification scheme) have mesostases which are feldspar normative with moderate to low amounts of normative quartz. Other chondrules (which are termed group B1 chondrules) have appreciable quartz in their mesostasis norms even though they contain olivine, which is indicative of considerable supercooling.

As many authors have pointed out, both olivine and mesostasis compositions show major changes during metamorphism $[6,7,24-26]$. Understanding the effects of metamorphism has proved important in our efforts to understand both meteorite formation and history on the parent body and thus the compositional changes to the chondrules accompanying metamorphism must also be in-

Table 1

An interpretation of the formation of the major chondrule groups in terms of the intensity of the heating event ${ }^{*}$ and subsequent crystallization

\begin{tabular}{lll}
\hline Chondrule group & Intensity of heating event & Cooling rate \\
\hline $\mathrm{A} 1^{*}$ & High, reduction and loss of volatiles during crystallization & Slow, phenocrysts and mesostasis at equilibrium \\
$\mathrm{A} 2$ & High, but slightly less reduction and volatile loss than A1 & Same as A1 \\
$\mathrm{A} 5^{\text {(a) }}$ & Low, no reduction or mass loss & Same as A1 \\
$\mathrm{B}^{+}$ & Low, no reduction or mass loss & Fast, considerable supercooling \\
\hline
\end{tabular}

'Intensity of heating event' refers to the chemical changes caused by the chondrule formation event. The major factor responsible for these was almost certainly temperature, but time spent at the peak temperature during formation, cooling rate, and absence of insulating materials (especially oxygen-rich dust) will also influence the effect of the heat pulse. "Includes some of the droplet chondrules of Kieffer [41], some of the non-porphyritic pyroxene chondrules of Gooding and Keil [42], the type I chondrules of McSween [16], metal-rich microporhyritic chondrules of Dodd [43], and the type 1A chondrules of Scolt and Taylor [17]. * Includes the poikilitic pyroxene and type IB chondrules of Scott and Taylor [17] and many of the type IAB chondrules of Jones [18.19]. ("There appear to be no previous observations of this chondrule group in unmetamorphosed meteorites. " Dodd's [44] 'lithic" or 'clastic' chondrules and Dodd's [43] metal-poor microporphyritic chondrules are included in this group, as are the type II chondrules of McSween [16], Scolt and Taylor [17] and Jones [30]. 
cluded in the chondrule classification. Placing the field boundaries so as to be of maximum value is very difficult and will no doubt improve as work progresses, especially as we extend it to enstatite and carbonaceous chondrites.

\subsection{Chondrule classification: Olicine composition}

The primary compositional parameters for chondrule olivine are the $\mathrm{CaO}$ and $\mathrm{FeO}$ contents (Fig. 1). The compositional boundaries we used for olivine in our earlier papers [6,7] (Fig. 1a) were placed so as to enclose existing data for a small group of meteorites, but an alternative and possibly preferable approach is to draw straight lines across the plot in the fashion shown in Fig. $1 \mathrm{~b}$. This results in a simpler scheme and fills the available $\mathrm{CaO}-\mathrm{FeO}$ space, especially the gap between groups $\mathrm{A} 3$ and $\mathrm{A} 4$, a region which, however, we note is poorly populated on the basis of currently available databases. We emphasize that the simplifications to our original graph do not result in the wholesale changing of classifications for previously classified chondrules; nor do they change the luminescence distinctions we have noted between the various chondrule groups.

We do not know the exact trajectory of the olivine compositions across the diagram during metamorphism, because compositional zoning as well as chondrule size and grain-size effects result in unique paths for each individual grain. The curved arrows in Fig. 1b describe some possibilities. The major trajectories seem to be $\mathrm{A} 1 \rightarrow \mathrm{A} 3$ $\rightarrow \mathrm{A} 4 \rightarrow \mathrm{A} 5, \mathrm{~A} 2 \rightarrow \mathrm{A} 5, \mathrm{~A} 1 \rightarrow \mathrm{A} 2 \rightarrow \mathrm{A} 5$ and $\mathrm{B} 1$ $\rightarrow \mathrm{B} 2 \rightarrow \mathrm{B} 3 \rightarrow \mathrm{A} 5$, but even $\mathrm{A} 1 \rightarrow \mathrm{A} 2 \rightarrow \mathrm{A} 3 \rightarrow$ $\mathrm{A} 4 \rightarrow \mathrm{A} 5$ is possible, although the latter trajectory is probably the least likely given the very narrow range of overlap in calcium contents between these two chondrule groups.

\subsection{Chondrule classification: Mesostasis composi- tion}

Fig. 2 shows the available data for Semarkona (type 3.0) chondrule mesostasis compositions. We have now completed three major studies of chondrule compositions for type 3 ordinary chondrites [26-28]. Each project was performed with differ-

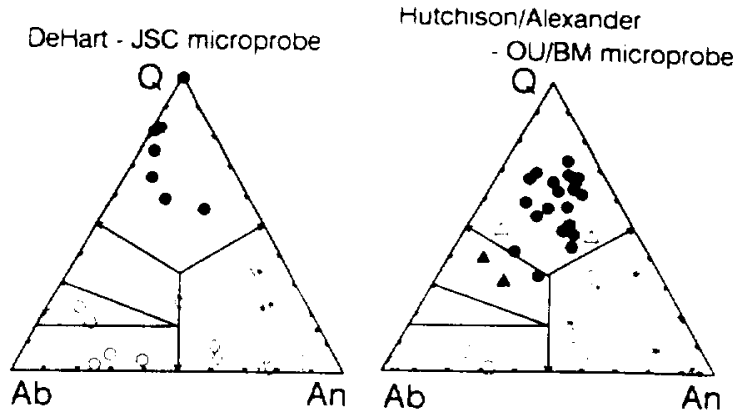

Lu Jie - AMNH microprobe
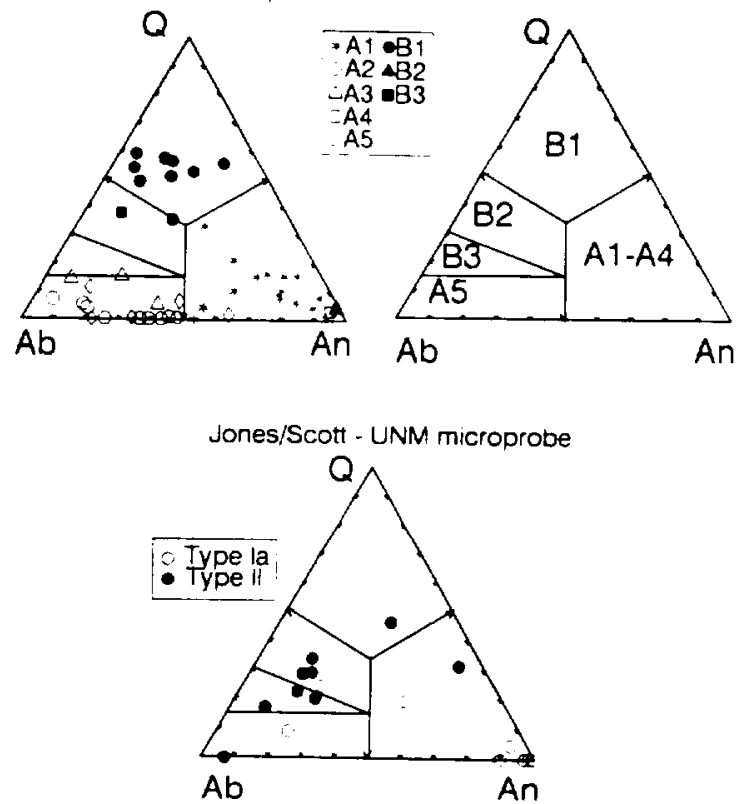

Fig. 2. Comparison of mesostasis compositions for Semarkona chondrules determined by a variety of analysts using different electron microprobes. J. DeHart with the Johnson Space Center microprobe $[6,25]$. R. Hutchison and C. Alexander with the British Museum and Open University microprobes [28] and L. Jie with the American Museum of Natural History microprobe $[12,26]$ produced very similar data, while the data obtained by $R$. Jones and E. Scolt with the University of New Mexico microprobe are quite different, reflecting their use of a broader analysis beam [22.28].

ent collaborators, and thereby different analysts and different electron microprobes, and each has either been published [6,7], is in press [28], or is about to be submitted [29]. The number of chondrules we have studied in detail is now nearly 200 and includes a wide selection of those present in our research samples, rather than a select subset 
picked on the basis of a variety of textural and mineralogical criteria. All our studies show that many chondrules in Semarkona (the group B1 chondrules) contain over (sometimes well over) $50 \mathrm{~mol} \%$ normative quartz when the data are projected onto the normative quartz-albiteanorthite ternary diagram. The Scott et al. [9] Semarkona data are also shown in Fig. 2 and clearly differ from the data from the other laboratories. The differences largely reflect differences in analytical technique, in turn reflecting differences in intended application. The literature data shown by Scott et al. [9] for 29 chondrules were obtained in an attempt to determine the composition of 'residual' melt and were thus collected using relatively large beam diameters in order to integrate glass and 'quench' crystals $[23,30]$. In contrast, the other analysts use smaller beam diameters confined to the glass (including crystal microlites which are not apparent in optical microscopy). We note that, under the latter application, the 'mesostasis' to be analyzed inevitably exhibits $\mathrm{CL}$, which can be used to iden- tify appropriate regions in the electron microprobe prior to analysis $[6,7,26,27]$.

For the few instances of glassy or cryptocrystalline chondrules a meaningful classification can usually be obtained by plotting a defocussed electron beam analysis of the entire chondrule, or an average of several regions of the chondrule, on the mesostasis plot. Such an application of the scheme cannot distinquish between some groups of chondrules, especially for the Al-A4 series which differ in their definitions largely in the mineral chemistry of olivine phenocrysts. Further work is needed on these rare chondrules, but we feel that it is appropriate to at least initially group them with other chondrules with which they at least share some chemical similarity.

\subsection{Facility of the compositional classification scheme}

It is worth detailing briefly the relative ease with which the various chondrule classification schemes can be applied. By applying the miner-

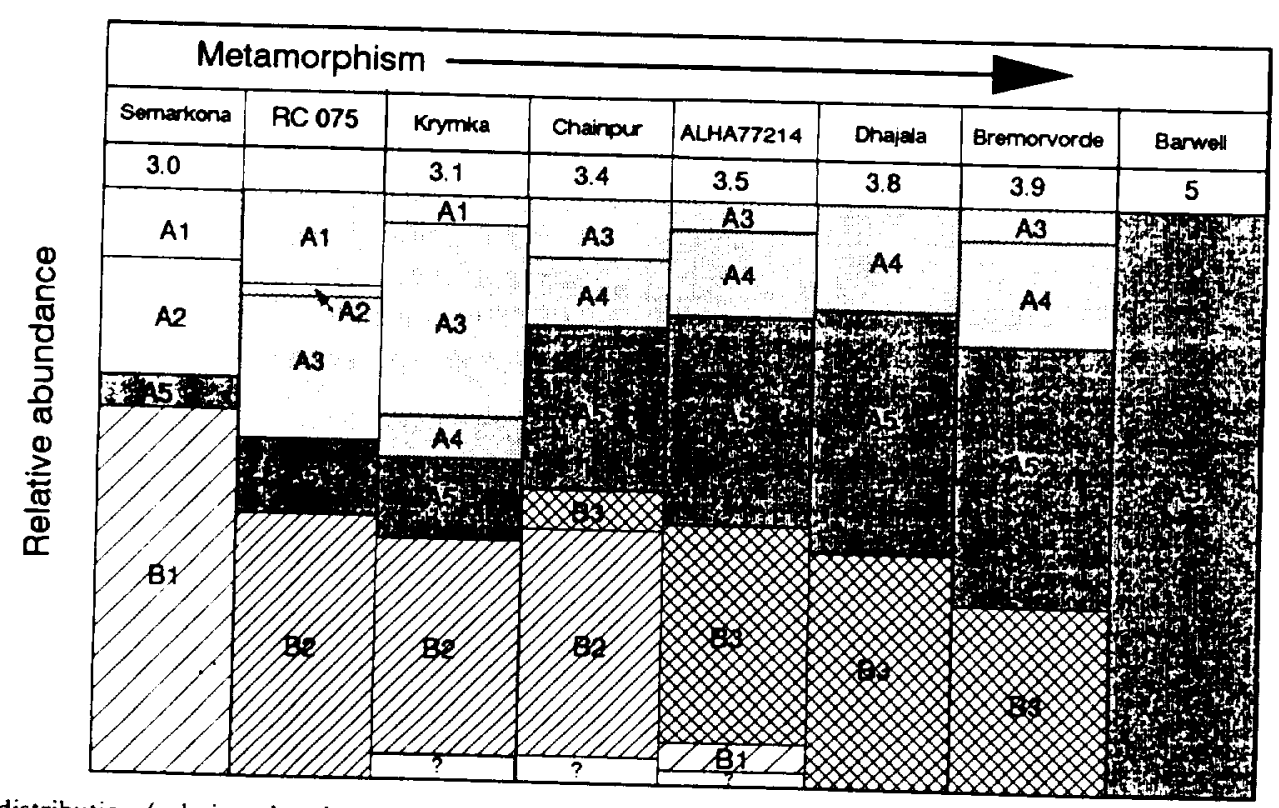

Fig. 3. The distribution (relative abundance by number) of chondrules over the compositional classes for six type 3 ordinary ordinary chondrites and Barwell (LS). The classifications were made from low-power photomicrographs of sections, using the CL to
determine the compositions of the major phases in the chention to compositional groups and, since composition and chondrules. It is possible to assign virtually all the chondrules in the sections conditions), it is possible to assign the especially therefore the chondrule group varies with metamorphism (as well as formation 
alogical-textural 'type' scheme to the chondrules separated from their host meteorite during one of our projects we were able to classify 5 out of 23 chondrules $[12,31]$, and in the light of recent changes introduced by Jones $[18,19]$ could still only classify a further 4 chondrules. The remaining chondrules simply did not display the large array of textural and mineralogical properties now included in the definitions of types IA to ILAB, whether the chondrules were examined by us or by our collaborators. When the compositional classification scheme is applied to chondrules in thin section $>95 \%$ of the chondrules were successfully classified in a number of different type 3 ordinary chondrites (Fig. 3).

Most of our studies have involved chondrules which have been physically removed from the meteorite and represent more challenging tests of the schemes since these are intended to be random samples of chondrules rather than subsets homogeneous in one or more properties. We have examined data for 107 chondrules from the studies of DeHart [26] and Lu [27]. Only 17 chondrules were either devoid of olivine or the olivine plotted outside the fields, while for only 8 chondrules were there no mesostasis data. It was possible to classify 100 chondrules when both parameters were used. In about two-thirds of the cases mesostasis alone would have enabled classification, while in about one-third of the cases olivine compositions are alone sufficient.

Perhaps the strongest difference between the two classification schemes lies in their major emphasis. Our proposed scheme classifies chondrules on the basis of major element chemistry of major chondrule phases (i.e., olivine and mesostasis). As such, it can be applied with little or no disagreement by anyone, including those with only limited experience with chondrules or with experience of the more qualitative aspects of chondrule petrographic description. The previous classification scheme was originally based largely on petrographic textures and was thus subjective and required a fair degree of previous knowledge of relative textures before it could be successfully applied. Later work added mineral chemistry to this scheme, but only as an adjunct to texture and only in an individual meteorite (i.e., Semarkona).
A key assumption in this scheme is that texture, to which petrographers were necessarily biased in the days before the wide availability of electron microprobes, must reveal more about the history and origin of chondrules than their major element chemistry. We argue that the availability of electron microprobes and the progressively increasing ease of their use, as well as increasing uncertainty in the basis of laboratory studies of the significance of chondrule texture, are good reasons to move to a more quantitative classification scheme.

\subsection{Pyroxene composition}

Although Huang et al. [29] were able to use olivine for the classification of 90 of their 107 chondrules, a few chondrules contain only olivine which is too fine grained to analyze or contain only pyroxene. Obviously one cannot simply plot pyroxene data on the olivine mineral chemistry graph (Fig. 1a) although, in analogy to glassy chondrules discussed above, the mesostasis compositions of these chondrules can be used to obtain an approximate classification in most cases. We anticipate that a pyroxene analog to Fig. 1 will eventually be added to the classification scheme, although the literature data on which to produce such a plot are at present scarce. Our present applications, however, are examinations of chondrule populations in the broadest sense, and for such general applications the scheme in its present form is sufficient.

\section{The petrographic classification of Roosevelt County 075}

While LL chondrites of petrologic type $<3.4$ are relatively common it has been only recently that a few $\mathrm{H}$ chondrites of type $<3.4$ have been reported. One of them is the heavily weathered Roosevelt County 075 [32]. Weathering and the lack of equilibration make classification uncertain, but it is probably an $\mathrm{H}$ chondrite. Weathering also makes it very difficult to assign a petrologic type. For example, removal of the weather- 
ing products by acid-washing increased the thermoluminescence sensitivity of RC075 by a factor of $\sim 7$, equivalent to a change in petrologic type estimate from 3.0 to 3.3 - a major difference. Since the compositional classification scheme reflects metamorphic effects as well as primary chondrule differences and can be applied to virtually all the chondrules present, it is well suited to the assignment of a petrologic type to RC075 by comparison with the chondrule populations of other meteorites of known petrologic type.

Cathodoluminescence properties of the phases in chondrules are simply related to composition, so that it is possible to assign all the chondrules in our $7 \times 5 \mathrm{~mm}$ section of RC075 to compositional classes using their CL. While not as finetuned as the quantitative classification by mineral chemistry, the use of luminescence permits rapid, reproducible classification of virtually all chondrules in a given thin section, and thus permits statistically significant sampling of chondrule populations. The apparatus used in the present application is a relatively simple commercially available attachment (a nuclide luminoscope) for an optical microscope and our operating conditions (e.g., for the electron beam) are typical for routine petrographic applications. Contrary to an apparently misunderstood personal communication cited by Scott et al. [9], we know of no case where minerals luminesce blue in the luminoscope $\mathrm{CL}$ apparatus and red in the electron microprobe, and we know of no physical way to achieve such a change in the $\mathrm{CL}$ activator. Steele has performed a detailed series of studies on the $\mathrm{CL}$ properties of meteoritic minerals using an electron microprobe [33] and we know of instances where his data differ from our own observations with a luminoscope.

The results of applying our classification scheme to the chondrules in RC075 are shown in Fig. 3, along with similar data for seven other ordinary chondrites $[6,7]$. The relative abundance of group B chondrules in RC075 is less than in the type 3.0 ordinary chondrite Semarkona, and comparable with the higher types, while the abundance of A5 chondrules is comparable to that in the type 3.1 chondrite Krymka and intermediate between that in Semarkona and Chain- pur (type 3.4). Most significantly the fraction of Al chondrules is very large and comparable (within error) to that of Semarkona, while the large number of group A3 chondrules is comparable only to Krymka. Although RC075 is a heavily weathered meteorite we do not believe that weathering has affected our observations; our previous observations on chondrules from other meteorite finds indicate that their chondrules have TL properties similar to those of unweathered meteorites of similar petrologic type. On the basis of these data RC075 would appear to be intermediate to Semarkona and Chainpur and comparable to Krymka in its petrologic type.

McCoy et al. [32] report means ranging from 0.07 to $7.2 \mathrm{~mol} \% \mathrm{Fa}$ and 0.11 to $0.36 \mathrm{wt} \% \mathrm{CaO}$ for olivine in six type 1A chondrules and 12.3-20.2 $\mathrm{mol} \% \mathrm{Fa}$ for five type Il chondrules in $\mathrm{RC} 075$. Four of the type IA chondrules resembled those of Semarkona in olivine composition $(<2 \mathrm{~mol} \%$ $\mathrm{Fa})$. Unlike the compositional classification scheme, which leads fairly simply to unambiguous petrologic type assignment, it seems difficult to assign RC075 to a petrologic type on the basis of olivine compositions and texture alone. It is quite difficult to learn anything from the relative abundance of type I and type II chondrules using purely textural definition of types in the fashion of McCoy et al. [25]-even if these could be determined for a significant proportion of the chondrules present-since they are independent of metamorphism. The compositional classification scheme provides one of the best methods for assigning the weathered and highly unequilibrated RC075 meteorite to a specific petrologic type, and suggests that it is type 3.1. Thermoluminescence sensitivity, which is directly related to $\mathrm{CL}$ intensity (blue wavelengths in particular), produces a similar result, but, as a bulk technique, is more affected by weathering. In any case, it is apparent that RC075 is the lowest petrologic type $\mathrm{H}$ chondrite presently known.

Some points to stress in favor of adopting the compositional classification scheme are that (1) the technique is feasible because major changes in the composition of chondrules occur with small changes in metamorphism, and (2) being comprehensive it is possible to make meaningful state- 
ments about the proportion of various chondrule groups.

\section{Brecciation in type 3 ordinary chondrites}

More than $40 \%$ of type 3 ordinary chondrites are known or strongly suspected to be breccias [34]. This figure is almost certainly an underestimate because research samples and sections tend to be very small, especially in comparison with normal hand specimens. It is possible to use some bulk properties, such as carbon content, olivine heterogeneity and thermoluminescence sensitivity, to estimate the mean degree of metamorphism experienced by the meteorite or at least to estimate the range of metamorphism exhibited by subregions of the meteorite. To assume that an individual chondrule in a breccia has been metamorphosed to exactly the same degree as the 'mean' meteorite is, however, a dangerous fallacy, and to assume that all meteorites are not brecciated until proven otherwise is a still greater fallacy.

Ngawi is a spectacular example of a brecciated chondrite with bulk properties (measured, for example, by induced thermoluminescence, carbon content and olivine heterogeneity) equivalent to petrologic type $\sim 3.7$. It is apparent that this meteorite is brecciated on the thin-section $(<1$ $\mathrm{cm}$ ) scale (Fig. 4). Scott et al. [35] published olivine composition data for both the brecciated ALHA 77278 and Ngawi and these data are superimposed on the fields which are supposed to correspond to the petrologic type of the host chondrite [9] in Fig. 5. Clearly the data for both meteorites spread across the entire diagram, indicating that a knowledge of the average petrographic type is a poor guide to expected chondrule composition in brecciated meteorites.

We obtained a CL mosaic of a thin section of Ngawi and classified all the chondrules in the section on the basis of their luminescence. A sketch of this section is shown in Fig. 4. It is apparent, as was also apparent in the chemical data (Fig. 5), that there is a great diversity of chondrules in this single section. The presence of A4, A5 and B3 chondrules is expected consider-
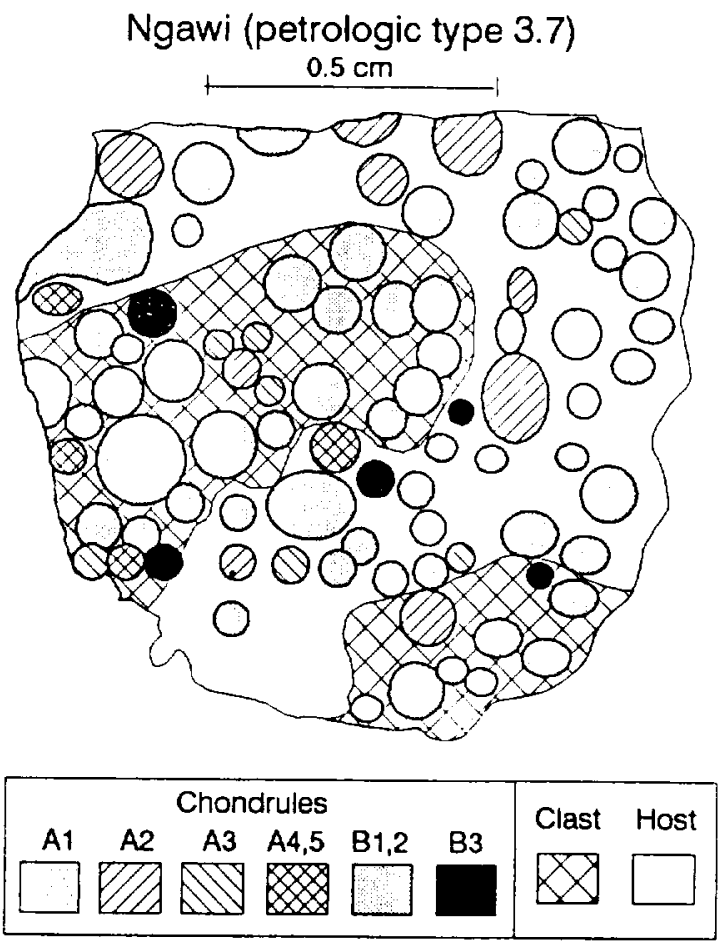

Fig. 4. Sketch of the texture of Ngawi with the chondrules assigned to compositional classes using their $\mathrm{CL}$ properties. Ngawi is heavily brecciated, containing low petrographic type clasts in a host matrix which is largely of high petrographic type but with chondrules of all compositional groups. According to most conventional criteria Ngawi is type 3.7 .

ing the average high petrologic type of the host meteorite, in analogy to the Dhajala meteorite (Fig. 3). However, the other chondrule groups. including group A1, A2 and B1,2 chondrules, are also present in significant numbers. In this the meteorite resembles the most 'unequilibrated' chondrites, such as Krymka, Semarkona, and RC 075 which was discussed above (Fig. 3).

On the basis of these data we find that setting apparent metamorphic boundaries for chondrules (Fig. 5) vastly oversimplifies the complexity of chondrules, especially in brecciated meteorites. On the basis of Fig. 5 one might expect all or most chondrules in a meteorite described as of some average petrologic type to exhibit very restricted chondrule compositions. In reality this is not necessarily the case in many meteorites. We prefer to describe chondrules only on the basis of 


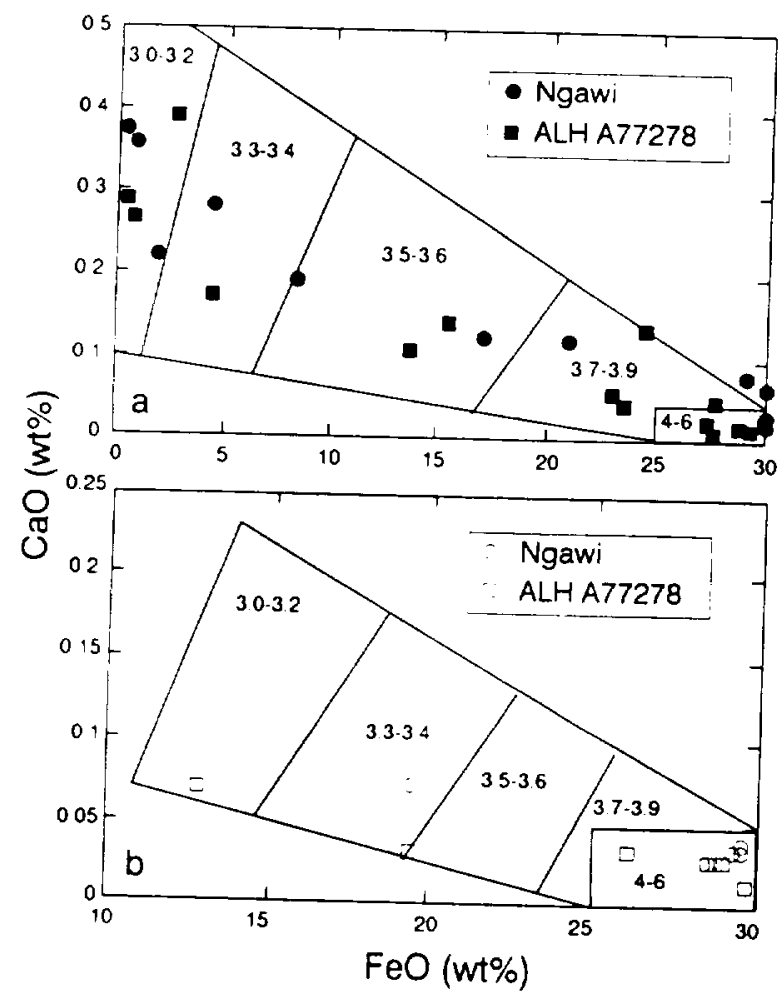

Fig. 5. CaO-FeO for chondrule olivines in Ngawi and ALHA 77278 [33]. The fields are those proposed by Scott et al. [9]. the upper figure describing how the composition of type IA chondrules varies with petrographic type; the lower diagram refers to type II chondrules. Clearly, knowing the type (IA, IB, IAB, II) of a chondrule and the petrologic type of the host meteorite does not describe the chondrule. At least $40 \%$, and probably most, of the type 3 chondrites are breccias and it is essential to characterize chondrules in terms of direct observations of the present chondrules.

the chemical compositions (or, as in this case, on the basis of the luminescence properties which derive from the mineral chemistry). Such a system makes no assumptions about the response of individual chondrules to 'bulk' rock metamorphism.

\section{The group A5 chondrules}

The presence of group A5 chondrules in highly unequilibrated meteorites such as Semarkona is of great interest [36]. The group A5 chondrules make up essentially $100 \%$ of the chondrules in equilibrated ordinary chondrites (petrologic types 5 and 6) and their abundance increases as a function of petrologic type within the unequilibrated type 3 ordinary chondrites (Fig. 3) and on this basis one might expect that they are a product of the high-temperature metamorphism of chondrules. Their presence in meteorites which have experienced little or no metamorphism and in significant numbers (about $15 \%$ of all chondrules in these meteorites) is thus an entirely new and unexpected discovery.

The group A5 chondrules stand out rather spectacularly in low-magnification CL images because of their bright blue mesostases and nonluminescent mineral grains. (By contrast the other common chondrules in the most unequilibrated chondrites have quite different luminescence properties, group Al,2 chondrules having bright yellow $\mathrm{CL}$ and group $\mathrm{Bl}$ chondrules being nonluminescent.) The $\mathrm{CaO}$ and $\mathrm{FeO}$ of the olivine in the AS chondrules of several LL chondrites covering a range of average petrologic types are shown in Fig. 6. Like the other chondrule groups, the olivines of group A5 chondrules appear to decrease in $\mathrm{CaO}$ and increase in $\mathrm{FeO}$ with increasing metamorphism, but much less than for the other group A chondrules and somewhat similar to the group B chondrules. The olivine heterogeneity (expressed as the coefficient of

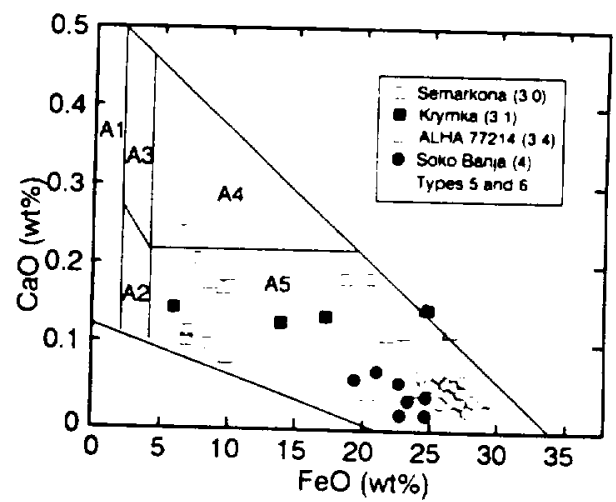

Fig. 6. The $\mathrm{CaO}-\mathrm{FeO}$ composition of olivine in the new group As chondrules. With metamorphism. $\mathrm{CaO}$ decreases and $\mathrm{FeO}$ increases. It would be a simple matter to subdivide the group to distiquish metamorphically derived subgroups. However. we refrain from doing so pending an examination of AS chondrules in further chondrites. (Data are from $[26,27,45,46]$.) 


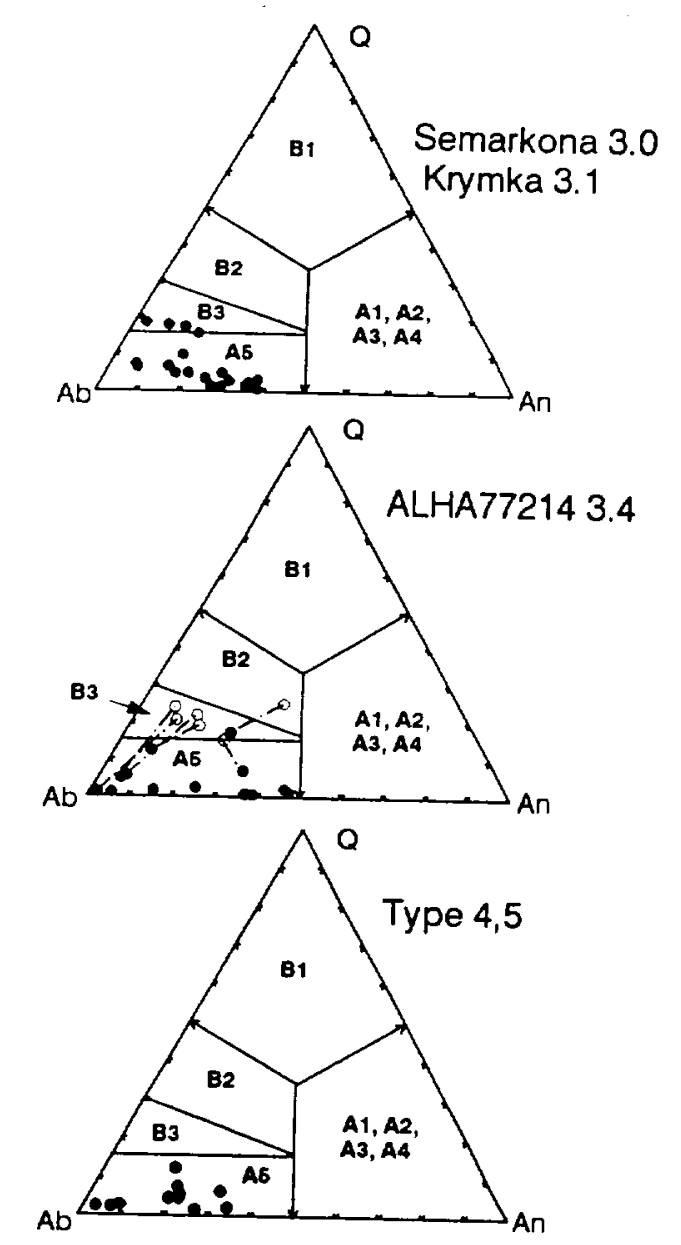

Fig. 7. Mesostasis compositions for group A5 chondrules. The data change little with petrographic type of the host meteorite. ALHA 77214 contains numerous chondrules which are compositionally zoned, being $\mathrm{B} 3$ at the center and $\mathrm{A} 5$ on the outside. Previous chondrule classification schemes would overlook this important property of this type 3.4 chondrite.

variation) increases from 5-20\% in types 3.0-3.1 to about $50 \%$ in type 3.5 , and then decreases to $<2 \%$ in types $4-6$. Low-Ca pyroxenes increase in $\mathrm{CaO}$ and $\mathrm{FeO}$ with metamorphic type in $\mathrm{As}$ chondrites. Mesostasis compositions (Fig. 7) are very similar in all $\mathrm{A} 5$ chondrules, with few readily delineated metamorphic trends. Our INAA data for group A5 chondrules from unequilibrated chondrites show them to have unfractionated bulk compositions relative to homogenized $\mathrm{Cl}$ chondrite.
The compositional classification scheme for chondrules does not, at present, differentiate between metamorphosed and unmetamorphosed A5 chondrules. Small differences certainly exist. Metamorphism causes homgenization and a small increase in the $\mathrm{FeO}$ content of the olivines, for instance. The olivines in group B1 and B3 chondrules show similar differences. However, these are small compared to the very large changes in mesostasis composition observed for group B1 chondrules but not observed for group $A 5$ chondrules. Future work might make it possible to subdivide the AS group, but this should be done with caution. It currently seems to us that the similarities between the group A5 chondrules in unequilibrated and equilibrated chondrites are more important than their differences.

The changes in compositional heterogeneity observed among the olivines and pyroxenes of group A5 chondrules suggests that the group A5 chondrules in Semarkona were not metamorphosed prior to emplacement in the meteorite, and the presence of A5 chondrules in Semarkona is not due to brecciation. Rather, we interpret this as meaning that the chondrule-forming process was capable of producing chondrules with mineral phase compositions resembling those of chondrules in equilibrated chondrites. Based on mineral chemistry we can make a number of interpretations about the chondrule-forming process. There are several lines of evidence (summarized by [37] and [38]) which suggest that group Al chondrules experienced higher temperatures during formation than other chondrules. We have previously argued that this also resulted in their volatile-poor and highly reduced compositions $[12,29]$. Conversely, the volatile-rich, oxidized group B1 chondrules apparently experienced lower peak temperatures during formation. The composition of the mesostasis relative to the olivine / pyroxene grains allows us to infer supercooling in the case of group $B$ chondrules but not in the case of group A chondrules, including A5 $[20-23,29]$. We suggest that the four chondrule groups in Semarkona represent three very different thermal histories. The group $\mathrm{A} 1$ and $\mathrm{A} 2$ chondrules appear to have formed at relatively high temperatures and did not experience super- 
cooling during subsequent crystallization, while the group B1 chondrules formed at lower temperatures but with supercooling. The group A5 chondrules may have formed at low temperatures, similar to group B1 chondrules, but did not experience supercooling (Table 1). We suggest that the cooling history of chondrules must have been independent of the intensity of the heating pulse which formed them and probably depended on local gas and dust density. Zoned mesostasis and sulfide- and metal-rich rims around group $A$ chondrules might imply an environment suitable for recondensation of volatiles for group A $[39,40]$. We note, however, that these are interpretations and we do not suggest classifying chondrules according to the presumed nature of their formation process or subsequent cooling history.

\section{The classification of chondrules: Some con- cluding remarks}

Classifications should be based on objective descriptions of the objects being classified, concentrating on the most fundamental properties of the objects (i.e., those of greatest importance with respect to origin and history). Most certainly they should not be model-dependent or dependent on assumptions about the original properties of the objects or what has happened to them since their formation, regardless of how sensible those assumptions may seem at any given moment in time. We suggest that the most fundamental way of describing a chondrule is in terms of the composition of its two major components, both of which reflect initial formation and metamorphism and both of which have been shown by a great many studies to provide important insights into chondrule history. It seems to us that to define groups of chondrules in terms of the composition of one phase and texture and then to adjust the compositional parameter depending on the petrographic type of the host chondrite is to make unreasonable generalizations about the original nature of the chondrule and its response to meta. morphism. It also seems a mistake to ignore brecciation, the influence of which is probably greatly underestimated. The response to meta- morphism of a given chondrule is, of course, very complicated and will depend on a great many factors, such as chondrule texture, chondrule size, the nature of adjacent phases, and the presence of cracks through which diffusion or other modes of transport could occur. Most serious of all, brecciation will cause chondrules of very different metamorphic histories to appear adjacent to each other in the same meteorite, and we believe that most, and probably all, type 3 ordinary chondrites are breccias.

It is certainly true that the compositional classification scheme needs further work. It took 150 years to arrive at the present situation, with considerable redundancy in each step and a great many small steps. Some of the initial criticisms of the compositional classification scheme are providing the first contribution to polishing the scheme, and are reflected in adjustments to the compositional boundaries shown in Fig. 1 b.

We end our paper by returning to the idea that a bad classification scheme can hinder rather than help our research efforts. McSween [16] introduced 'type I' and 'type II' during his studies of $\mathrm{C} 2-3$ chondrites, meaning essentially chondrules with $\mathrm{FeO}$-rich and $\mathrm{FeO}$-poor olivines. $\mathrm{He}$ rightly recognized the importance of the redox state of chondrules in our efforts to understand chondrules and chondrites. However, in applying the scheme to ordinary chondrites, olivine composition and texture have become confused, since textures change little but compositions change considerably during metamorphism. Thus we have potentially confusing 'anomalies', such as type I chondrules being originally defined, and now widely known as, FeO-poor when in fact $\mathrm{FeO}$ poor type I chondrules are very rare and present in only a handful of ordinary chondrites. Thus when a serious study of metamorphism of ordinary chondrite chondrules was undertaken by McCoy et al. [25] they had to substitute the well-known and commonly understood definition of 'type I' with a purely textural definition. This point alone underlines the need for a better classification scheme. We argue instead that we should anchor chondrule studies (and classification) in the most fundamentally important and most objectively determined properties of the 
chondrules, namely the chemical composition of their major phases as determined by electron microprobe or luminescence techniques.

\section{Acknowledgements}

We are grateful to John Wood, Robert Hutchison, and Rhian Jones, Martin Prinz and Conel Alexander, for their reviews of this manuscript, and to Ed Scott and Rhian Jones for discussions of chondrule classification and for loaning us their thin sections. We also wish to thank Steve Symes for help with mansucript preparation, Walter Manger for loan of the cathodoluminescence apparatus, and Gary Lofgren, Martin Prinz, Robert Hutchison, Lu Jie, John DeHart, Andrew Morse, Conel Alexander, Mike Lipschutz and Jeff Grossman for several years of rewarding collaborative research on chondrules. This work was funded by NASA grant NAGW 3519. [UC]

\section{References}

[1] J.F. Kerridge and M.S. Matthews, Meteorites and the Early Solar System, 1269 pp., University of Arizona Press, Tucson, 1988.

[2] E.A. King, Chondrules and their Origins, 377 pp., Lunar Planet. Sci. Inst., Houston, 1983.

[3] J.A. Wood, Meteoritic constraints on processes in the solar nebula, in: Protostars and Planets II, D.C. Black and M.S. Matthews, eds., pp. 687-702, University of Arizona Press, Tucson, 1985.

[4] C.M. Wai and J.T. Wasson, Nebular condensation of moderately volatile elements and their abundances in ordinary chondrites, Earth Planet. Sci. Lett. 36, 1-13, 1977.

[5] E. Anders, Critique of 'Nebular condensation of moderately volatile elements and their abundances in ordinary chondrites' by C.M. Wai and J.T. Wasson, Earth Planet. Sci. Lett. 36, 14-20, 1977

[6] J.M. DeHart, G.E. Lofgren, J. Lu, P.H. Benoit and D.W.G. Sears, Chemical and physical studies of chondrites $\mathrm{X}$ : Cathodoluminescence studies of metamorphism and nebular processes in type 3 ordinary chondrites, Geochim. Cosmochim. Acta 56. 3791-3807, 1992.

(7) D.W.G. Sears, J. Lu, P.H. Benoit, J.M. DeHart and G.E. Lofgren, A compositional classification scheme for meteoritic chondrules, Nature 357, 207-211, 1992.
[8] J.A. Wood, Chondritic meteorites and the early solar nebula, Annu. Rev. Earth Planet. Sci. 16, 53-72, 1988.

[9] E.R.D. Scott. R.H. Jones and A.E. Rubin, Classification, metamorphic history, and pre-metamorphic composition of chondrules, Geochim. Cosmochim. Acta 58, 1203$1209,1994$.

[10] J.A. Wood, A. Hashimoto and B.B. Holmberg, Chondrules as near-equilibrium assemblages that formed in fractionated systems. Lunar Planet. Sci. XX, 1215-1216, 1989.

[11] A. Kracher, Why do some chondrules have high primary FeO contents?, Lunar Planet. Sci. XVI, 467-468, 1985.

[12] J. Lu, D.W.G. Sears, B. Keck. M. Prinz, J.N. Grossman and R.N. Clayton, Semarkona type I chondrules compared with similar chondrules in other classes, Lunar Planet. Sci. XIII, 720-721, 1990.

[13] H. Nagahara, I. Kushiro and B.O. Mysen, Evaporation of olivine: Low pressure relations of the olivine system and its implication for the origin of chondritic components in the solar nebula, Geochim. Cosmochim. Acta 58, 19511964. 1994

[14] A. Tsuchiyama, H. Nagahara and I. Kushiro, Volatilization of sodium from silicate melts and its application to the formation of chondrules, Geochim. Cosmochim. Acta 45, 1345-1367, 1981.

[15] A. Hashimoto, Evaporation metamorphism in the early solar nebula-evaporation experiments on the melt $\mathrm{FeO}$ $\mathrm{MgO}-\mathrm{SiO}_{2}-\mathrm{CaO}-\mathrm{Al}_{2} \mathrm{O}_{3}$ and chemical fractionations of primitive materials, Geochem. J. 17, 111-145, 1983.

[16] H.Y. McSween, Jr., Chemical and petrographic constraints on the origin of chondrules and inclusions in carbonaceous chondrites, Geochim. Cosmochim. Acta 41, $1777-1790,1977$.

[17] E.R.D. Scott and G.J. Taylor, Chondrules and other components in $\mathrm{C}, \mathrm{O}$, and $\mathrm{E}$ chondrites: similarities in their properties and origins, Proc. Lunar Planet. Sci. Conf. 14th, B275-B286, 1983.

[18] R.H. Jones, Classification of porphyritic, pyroxene-rich chondrules in the Semarkona ordinary chondrite, Lunar Planet. Sci. XXIII, 629-630, 1992.

[19] R.H. Jones, Petrology of FeO-poor, porphyritic pyroxene chondrules in the Semarkona chondrite, Geochim. Cosmochim. Acta, in press, 1994.

[20] M. Kimura and K. Yagi, Crystallization of chondrules in ordinary chondrites, Geochim. Cosmochim. Acta 44. 589-602, 1980.

[21] M. Blander, H.N. Planner, K. Keil and L.S. Nelson, The origin of chondrules: Experimental investigation of metastable liquids in the system $\mathrm{Mg}_{2} \mathrm{SiO}_{4}-\mathrm{SiO}_{2}$. Geochim. Cosmochim. Acta 40, 889-896, 1976

[22] G.E. Lofgren and W.J. Russell. Dynamic crystallization of chondrule melts of porphyritic and radial pyroxene composition, Geochim. Cosmochim. Acta 50, 1715-1726. 1986.

[23] R.H. Jones and E.R.D. Scott, Petrology and thermal history of type IA chondrules in the Semarkona (LL3.0) 
chondrite, Proc. Lunar Planet. Sci. Conf. 191h, 523-536, 1989.

[24] R.T. Dodd, W.R. Van Schmus and D.M. Koffman. A survey of the unequilibrated ordinary chondrites, Geochim. Cosmochim. Acta 31, 921-951, 1967.

[25] T.J. McCoy, E.R.D. Scott, R.H. Jones, K. Keil and G.J. Taylor, Composition of chondrule silicates in LL3-5 chondrites and implications for their nebular history and parent body metamorphism, Geochim. Cosmochim. Acta 55. $601-619,1991$.

[26] J.M. DeHart. Cathodoluminescence and microprobe studies of the unequilibrated ordinary chondrites, Ph.D. Thesis, Univ. Arkansas, Fayetteville, 1989.

[27] J. Lu, The physical and chemical studies of chondrules from type 3 ordinary chondrites, Ph.D Thesis, Univ. Arkansas, Fayetteville, 1992.

[28] D.W.G. Sears, A.D. Morse, R. Hutchison, R.K. Guimon, J. Lu, C.M.O'D. Alexander, P.H. Benoit, 1. Wright, C.T. Pillinger, T. Xian and M.E. Lipschutz, Metamorphism and aqueous alteration in low petrologic type ordinary chondrites, Meteoritics 30, in press, 1995.

[29] S. Huang, J. Lu, M. Prinz, M.K. Weisberg, P.H. Benoit and D.W.G. Sears, Chondrules: Their diversity and the role of open-system processes during their formation, Icarus, in prep., 1995.

[30] R.H. Jones, Petrology and mineralogy of type 11 chondrules in Semarkona (LL3.0): Origin of closed-system fractional crystallization, with evidence for supercooling. Geochim. Cosmochim. Acta 54, 1785-1802, 1990.

[31] J. Lu, D.W.G. Sears, P.H. Benoit, M. Prinz and M.K. Weisberg, Related compositional and cathodoluminescence trends in chondrules form Semarkona, Meteoritics 26, 317, 1991.

[32] T.J. McCoy, K. Keil, R.D. Ash, A.D. Morse, C.T. Pillinger, R. Wieler, T.K. Mayeda, R.N. Clayton, P.H. Benoit, D.W.G. Sears, I. Casanova, D.W. Muenow, C.B. Moore, C.F. Lewis and I.E. Wilson, Roosevelt County 075: A petrologic, chemical and isotopic study of the most unequilibrated known $\mathrm{H}$ chondrite, Meteoritics 28 , 681-691, 1993.

[33] I. Steele, Mineralogy of meteorites revealed by cathodoluminescence, in: Spectroscopic Characterization of Minerals and their Surfaces. L.M. Coyne, S.W.S. McKeever and D.F. Blake, eds., Am. Chem. Soc. Symp. Ser. 415. $150-164$.

[34] D.W.G. Sears, F.A. Hasan. J.D. Batchelor and J. Lu.
Chemical and physical studies of type 3 chondrites XI: metamorphism. pairing, and brecciation of ordinary chondrites, Proc. Lunar Sci. Conf. 2lih, 493-512, 1991.

[35] E.R.D. Scott, T.J. McCoy and K. Keil, Post-metamorphic brecciation in type 3 ordinan chondrites, Lunar Planet. Sci. XXIV, 1267-1268, 1993.

[36] S. Huang, P.H. Benoit and D.W.G. Sears, Group A5 chondrules in ordinary chondrites: their formation and metamorphism. Lunar Planet. Sci. XXV. 573-574, 1994.

[37] R.H. Hewins, Experimental studies of chondrules, in: Meteorites and the Early Solar System. J.F. Kerridge and M.S. Matthews, eds., pp. 660-679. University of Arizona Press, 1988.

[38] G.E. Lofgren, Dynamic crystallization of chondrule melts of porphyritic olivine composition: Texture experimental and natural, Geochim. Cosmochim. Acta 53, 461-470, 1989.

[39] S. Huang, P.H. Benoit and D.W.G. Sears, Metal and sulfide in Semarkona chondrules and rims: Evidence for reduction, evaporation and recondensation during chondrule formation, Meteoritics 28. 367-368, 1993.

[40] S. Matsunami, K. Ninagawa. S. Nishimura, N. Kubono, I. Yamamoto, M. Kohata, T. Wada. Y. Yamashita, J. Lu, D.W.G. Sears and H. Nishimura, Thermoluminescence and compositional zoning in the mesostasis of a Semarkona group Al chondrule and new insights into the chondrule-forming process, Geochim. Cosmochim. Acta 57, 2102-2110, 1992.

[41] S.W. Kieffer, Droplet chondrules. Science 189, 333-340, 1975.

[42] J.L. Gooding and K. Keil, Relative abundances of chondrule primary textural types in ordinary chondrites and their bearing on conditions of chondrule formation. Meteoritics 16, 17-43, 1981.

[43] R.T. Dodd, Compositions of droplet chondrules in the Manych (L3) chondrite and the origin of chondrules, Earth Planet. Sci. Lett. 40, 71-82, 1978.

[44] R.T. Dodd, Meteorites: A Petrologic Chemical Synthesis, Cambridge University Press. 1981.

[45] J.L. Gooding. Petrogenetic properties of chondrules in unequilibrated $\mathrm{H}-$, L-, and LL-group chondritic meteorites. Ph.D Thesis, Univ. New Mexico, Albuqerque. 1979.

[46] J.V. Heyse. The metamorphic history of LL-group ordinary chondrites, Earth Planet. Sci. Lett. 40, 365-381, 1978. 